\title{
Predicting the Outcomes of Transcatheter Aortic Valve Prosthesis Implantation Based on the Finite Element Analysis and Microcomputer Tomography Data
}

DOl. 10.17691/stm2016.8.1.11

Received June 30, 2015

E.A. Ovcharenko, Research Fellow, Laboratory of Novel Biomaterials, Department of Experimental and Clinical Cardiology';

K.U. Klyshnikov, Research Fellow, Laboratory of Novel Biomaterials, Department of Experimental and Clinical Cardiology';

G.V. Savrasov, DSc, Professor, Department of Biomedical Technologies²;

A.V. Batranin, Engineer-Researcher ${ }^{3}$;

V.I. Ganykov, MD, DSc, Professor, Head of the Laboratory of Interventional Methods of Atherosclerosis Diagnosing and Treatment";

A.N. Kokov, MD, PhD, Head of the Department of X-ray Computed Tomography';

D.V. Nushtaev, Engineer, Department of Design and Engineering Analysis4;

V.Y. Dolgov, Junior Researcher, Laboratory of Novel Biomaterials, Department of Experimental and Clinical Cardiology';

Y.A. Kudryavtseva, DSc, Head of the Department of Experimental and Clinical Cardiology';

L.S. Barbarash, MD, DSc, Academician of the Russian Academy of Sciences, Chief Researcher ${ }^{1}$

${ }^{1}$ Scientific Research Institute of Complex Problems of Cardiovascular Diseases, 6 Sosnovy Blvd., Kemerovo, 650002, Russian Federation;

2Bauman Moscow State Technical University, 5, Bldg. 1, 2-ya Baumanskaya St., Moscow, 105005, Russian Feferation; ${ }^{3}$ National Research Tomsk Polytechnic University, 30 Lenin Avenue, Tomsk, 634050, Russian Feferation;

4"TESIS" Company, 18 Yunnatov St., Office 705, Moscow, 127083, Russian Federation

The aim of the investigation is to assess the possibility of predicting the outcome of transcatheter aortic valve prosthesis implantation in real clinical practice on the basis of patient-specific modeling.

Material and Methods. Modeling of transcatheter bioprosthesis implantation was carried out based on clinical data of a patient aged 72 years. Multispiral computed tomography (CT) was performed before and after the operative intervention. Reconstruction of aorta and valvular apparatus geometry was done on the basis of obtained slices using computer-aided design (CAD). With the help of microcomputer tomography and a series of projection images and mathematical algorithms a 3D-model of the frame was reconstructed, on which a 3D-mesh from 17,000 cubic (C3D8)-elements was built. Simulation of the system component interaction was conducted using a finite element method involving a number of successive steps: preliminary balloon dilatation — frame compression in the catheter — releasing the frame from the catheter. To evaluate the accuracy of modeling results compared to the CT data of the patient with the implanted bioprosthesis a proprietary algorithm was developed using MATLAB R2014a software (The MathWorks, USA). Arrays of points corresponding to the center of the supporting frame beams, obtained in 11 orthogonal sections, were used as input data.

Results. Peculiarities of patient-specific approach to modeling the implantation of transcatheter CoreValve bioprothesis by means of the developed bioinformation algorithm has been analyzed. We managed to achieve a high convergence of simulation and CT data for the frame inflow area (the difference of the simulation results for the first three layers did not exceed $4 \%$ ). Comparison of the results in terms of the annulus area has also demonstrated a high convergence: the identity amounted to more than $90 \%$ for the inflow and middle zones of the supporting frame.

Conclusion. The highest level of detail, including calcium conglomerates modeling, as well as a comprehensive description of nonlinear elements of the system under study accurately reproduces the process of implantation of the transcatheter aortic valve prosthesis.

Key words: patient-specific modeling; finite element method; transcatheter aortic valve prosthesis; implantation modeling.

For contacts: Kirill U. Klyshnikov, e-mail: klyshnikovk@gmail.com 
Transecatheter implantation, first performed by Alain Cribier a decade ago, has became currently a new standard of aortic stenosis correction for inoperable patients and patients with a high perioperative risk [1]. A clinical experience accumulated during the following years allowed, on the one hand, confirmation of the efficacy of this method, and on the other hand, revealing some of its drawbacks: e.g. the risk of significant regurgitation directly correlating with an increase of mortality rate [2, 3]. The main cause of such complication, as shown in a number of investigations, is deformation of prosthesis frame: its ellipticity, dimension discrepancy and spacial configuration [4,5]. The data obtained has clearly demonstrated the importance of in-depth study of patient's anatomy and thorough preoperative planning of transcatheter implantation procedure, which promoted the development of predicting systems and the accompanying technologies to assist interventions [6].

In recent years finite element method (FEM) became widely used as a tool for simulating the work of biotechnical systems [7]. Besides, the development of computed tomography systems led to the emergence of a new specialized branch - patient-specific computer modeling [8]. This approach turned out to be the most perspective in solving the tasks of predicting transcatheter implantation of aortic valve prosthesis [9, 10]. On the other hand, the patient-specific approach requires a thorough preparation and initial data acqisition as their total error (resolution of computed and microcomputed tomography, quality of the mesh of finite elements of aorta and calcium conglomerates, material models and general settings of the system) may result in significant divergence of the predicted and actual results. Unfortunately, there are few publications in the literature, devoted to the effect of the described components on the quality and validity of simulation. Moreover, simplicity of the approach to the assessment of the differencies in the results of modeling and clinical data is still enhanced by the application of the simplified models of physical and mechanical characteristics of the objects under study [3, $11,12]$. All these considerations make the possibility of a wide application of this method in real clinical practice rather doubtful and require its verification.

The aim of the investigation is to assess the possibilities of a patient-specific simulation method for predicting the outcome of implantation of the transcatheter aortic valve prosthesis in real clinical practice.

Materials and Methods. Implantation of transcatheter bioprosthesis was modeled based on clinical data of patient I., 72 years, the informed consent from which to perform the investigation has been obtained. The following diagnosis has been established to the patient: "atherosclerotic stenosis of aorta, IV degree calcinosis, arterial hypotension stage III, left ventricular (LV) hypertrophy". Preoperative echoCG showed normal LV contractility, critical calcified stenosis of aortic valve with a gradient of $100 \mathrm{~mm} \mathrm{Hg}$, marked LV myocardium hypertrophy (up to $2 \mathrm{~cm}$ ). During operative intervention a $29 \mathrm{~mm}$ CoreValve (Medtronic, USA) bioprosthesis of aortic valve was implanted using transfemoral delivery system (Figure 1).

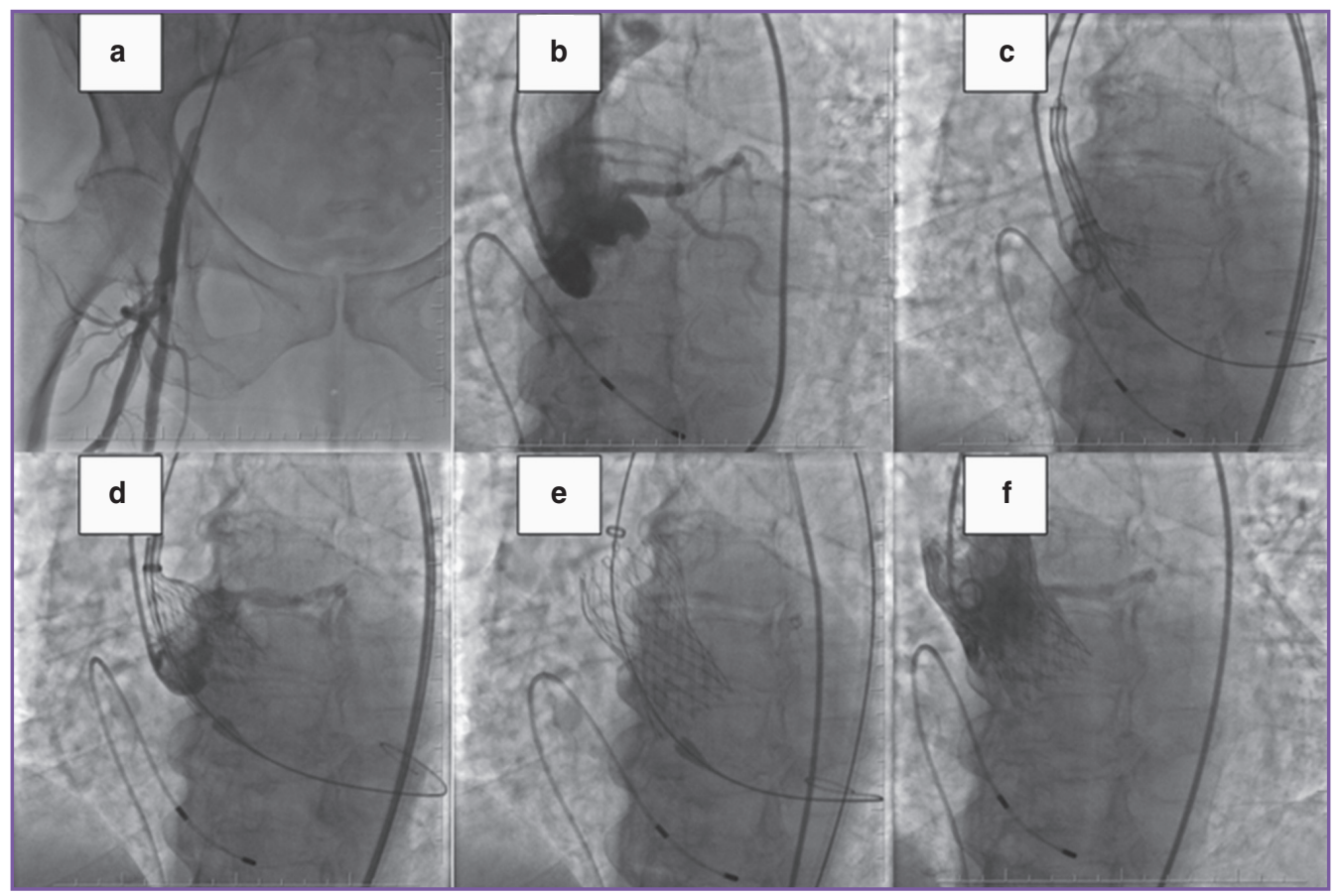

Figure 1. Stages of implantation of transcatheter CoreValve bioprosthesis: (a) visualization of aortoiliac and femoral segments; (b) aortography with visualization of aortic valve zone; (c) $-(e)$ release of the bioprosthesis from the delivery system; (f) control angiography 


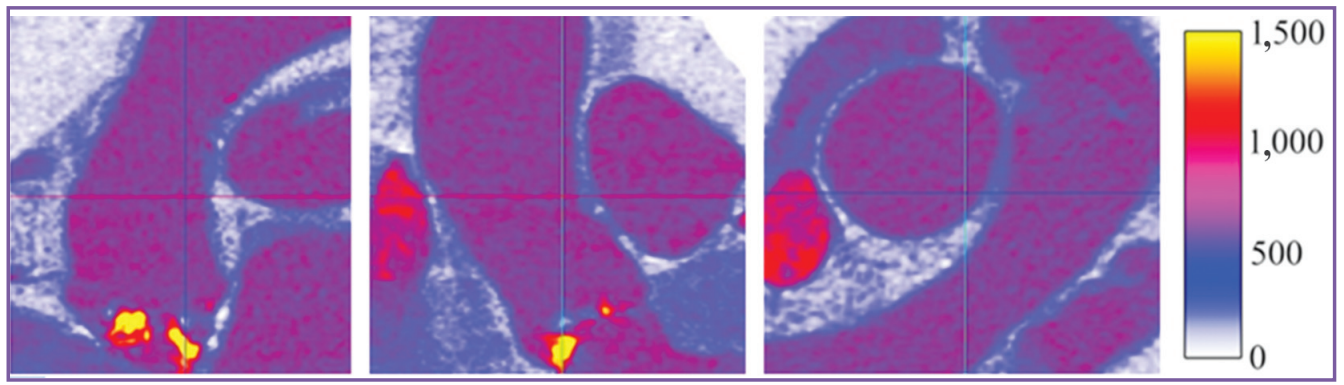

Figure 2. Results of multispiral computed tomography of the patient before the implantation of transcatheter CoreValve bioprosthesis

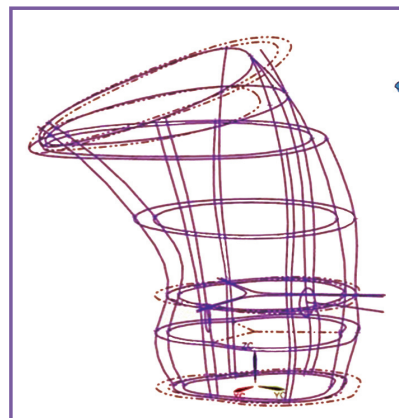

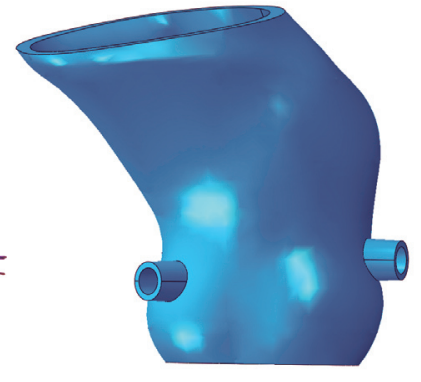

b



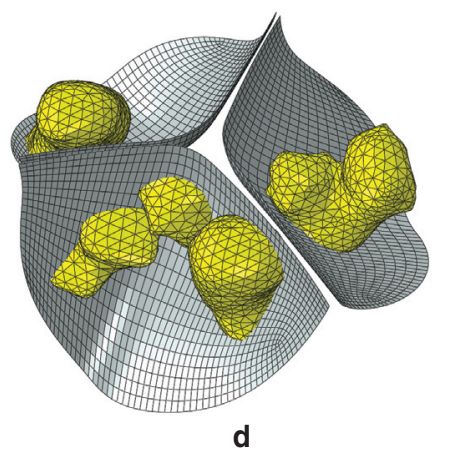

d

Figure 3. Successive stages of aorta reconstruction: (a) creation of splines on the basis of the obtained slice; (b) solid body; (c) finite element mesh; (d) leaflet apparatus and calcium conglomerates

Control angiography demonstrated a correct valve position, absence of regurgitation, impairment of atrioventricular conduction and compromised coronary blood flow. Operative intervention resulted in the decrease of the gradient to $5 \mathrm{~mm} \mathrm{Hg}$ and absence of clinical complications.

Prior the operation multispiral computed tomography (MSCT) was performed using Somaton Sensation 64 (Siemens, Germany) tomograph. Scanning was in a craniocaudal direction. MSCT main characteristics were as follows: field of vision (FOV) of $200 \mathrm{~mm}$, pitch of $0.7 \mathrm{~mm}$, tube voltage of $120 \mathrm{kV}$, ECG synchronization, slice thickness of $1 \mathrm{~mm}$. Nonionic contrast agent Ultravist at iodine concentration of $370 \mathrm{mg} / \mathrm{ml}(1-1.5 \mathrm{ml} / \mathrm{kg}$ of body mass) was used for intravenous introduction. Image reconstruction was performed by means of Amira software (FEl, Germany). Application of greyscale gradient coloration spectrum enabled clear visualizatin of aorta wall and calcium deposits (Figure 2).

On the basis of the slices obtained and using computer-aided design (CAD) program (UGS NS 8.0; Siemens, Germany) reconstruction of aorta geometry and valvular apparatus was carried out with the following generation of the finite element mesh running the program ABAQUS/CAE (Dassault Systems, France): 29,000 cubic (C3D8)-elements for aorta and 2,800 S4elements for the valve leaflet apparatus (Figure 3 ).

As a rule, heavy aortic stenosis is associated with calcinosis of the leaflet apparatus and a typical pattern of the given lesion is described in the literature [13]. But inspite of the marked pathology, it is worth to keep in mind, that before the implantation of this kind of prostheses, balloon valvuloplasty is always performed to the patients - and in this case it is reasonable to use a limited number of models of calcinates having the largest size and density. Calcium conglomerate model was built using tetrahedral mesh from 7,000 C3D4-elements and the given raidiographic density of the material by means of Amira program tools (See Figure 3).

Though MSCT is not included into the standard protocol of the post-operative examination [10], our patient agreed to undergo this procedure in order to assess the position of the implanted bioprosthesis. On the basis of the obtained data the model of the implanted prosthesis frame was reconstructed with the help of Amira program (Figure 4).

To conduct FEM simulation, the model of the valve frame was reconstructed by microcomputer tomography on the basis of the bioprosthesis selected for implantation. The work was done using the research tomographic system at Tomsk Polytechnic University (Figure 5).

The system consisted of the following main components:

X-ray tube MXR-451HP/11 (Comet, Switzerland) with accelerating voltage range of $20-450 \mathrm{kV}$, minimal focal spot size of $0.4 \mathrm{~mm}$, power up to $500 \mathrm{~W}$;

flat panel X-ray detector XRD 1622 AP (PerkinElmer, 


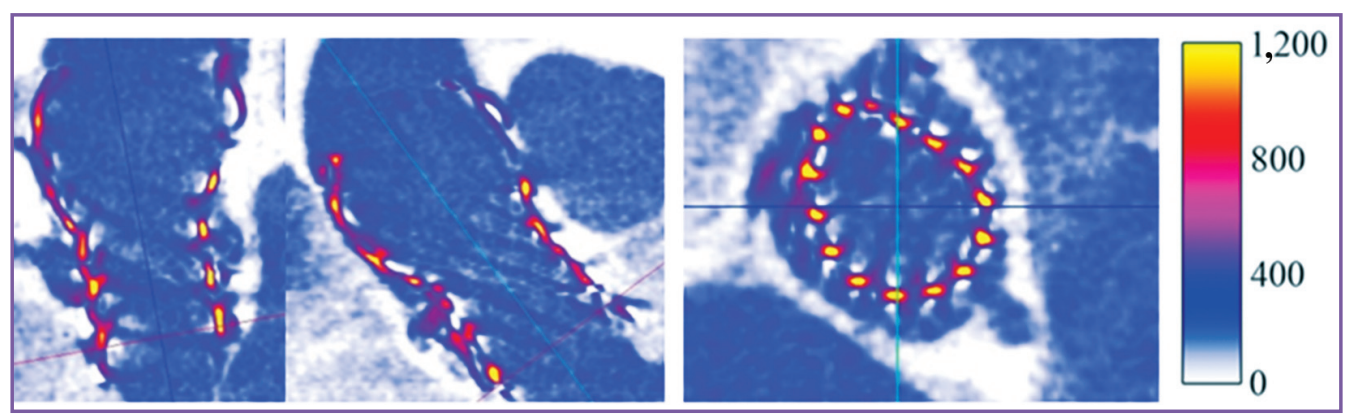

Figure 4. Results of multispiral computed tomography of the patient after the implantation of transcatheter CoreValve bioprosthesis

USA) with $0.2 \mathrm{~mm}$ pixel size, $2048 \times 2048$ pixel image size, 16-bit dynamic range;

mechanical manipulator with the feasibility of rotation and longitudinal movement of the examined object, positioning accuracy of $0.01^{\circ}$ and $0.01 \mathrm{~mm}$, respectively.

Tomographic scanning modes were chosen based on the sizes and radiographic density of the object: voltage of $200 \mathrm{kV}$, current of $2.7 \mathrm{~mA}$, copper filter thickness of $0.5 \mathrm{~mm}$, frame exposure of $1 \mathrm{~s}$, angular pitch of $0.4^{\circ}$, number of projections of 900 , voxel size of $64.4 \mu \mathrm{m}$.

Using the scanning data on the basis of projection image series and mathematical algorithms a 3D-model of the frame was reconstructed, which helped to build 3Dmesh from 17,000 C3D8-elements (Figure 6). The reconstruction of the leaflet apparatus could not be done due to the great difference in the radiological densities of the material of the frame and valvular apparatus. The properties of the Nitinol material model [14] were superimposed on the obtained finite element mesh according to the experimental data of modeling CoreValve frame compression and its following verification, presented by Tzamtzis et al.

During the actual procedure of prosthesis implantation the biotechnical system represents a complex of interacting objects "prosthesis-aorta-delivery system". In this connection, a simplified model of the delivery system, describing the border conditions for the implanted prosthesis frame, was reconstructed (Figure 7). This model contained two major limiting contours: internal contour (5,000 M3D4-elements) is static, external one (5,000 M3D4-elements) is variable, the general construction corresponded to the main components of the delivery system in the implantation zone [15]. The model had also a catheter for a guidewire, but it did not actually paticipate in simulation, as the function of the internal limitation was fulfilled by the internal contour, comprising a cylinder, which consisted of the sum of the valvular apparatus compressed volumes and a catheter for a guidewire.

For a more complete modeling of the implantation

process a simulation of balloon dilatation of the calcified leaflet apparatus - valvuloplasty - was preliminary performed using an additional balloon, representing a surface with the following properties: wall thickness of $0.15 \mathrm{~mm}$, Young's modulus of $290 \mathrm{MPa}$, Poisson coefficient $v=0.3$. Pressure of $0.2 \mathrm{MPa}$ was used to inflate the balloon. Once the balloon opened completely, pressure was reduced to zero. The obtained leaflet geometry, calcinates and aorta root were used for further interaction of these components with the supporting frame of the CoreValve prosthesis.

In order to stabilize the dynamic component of the system and their contacts after the interaction of the prosthesis with the aorta root, an artificial component for viscous-elastic damping was added, the energy contribution of which did not exceed $0.8 \%$ of the total system energy (Figure 8). To simulate the interaction of all system elements a paired softened contact description (penalty method) with the coefficient of friction $\mu=0.2$ was used [16]. The interaction "balloon-supporting frame" occurred without friction and interpenetration of the objects (hard-contact). In the experiments the 


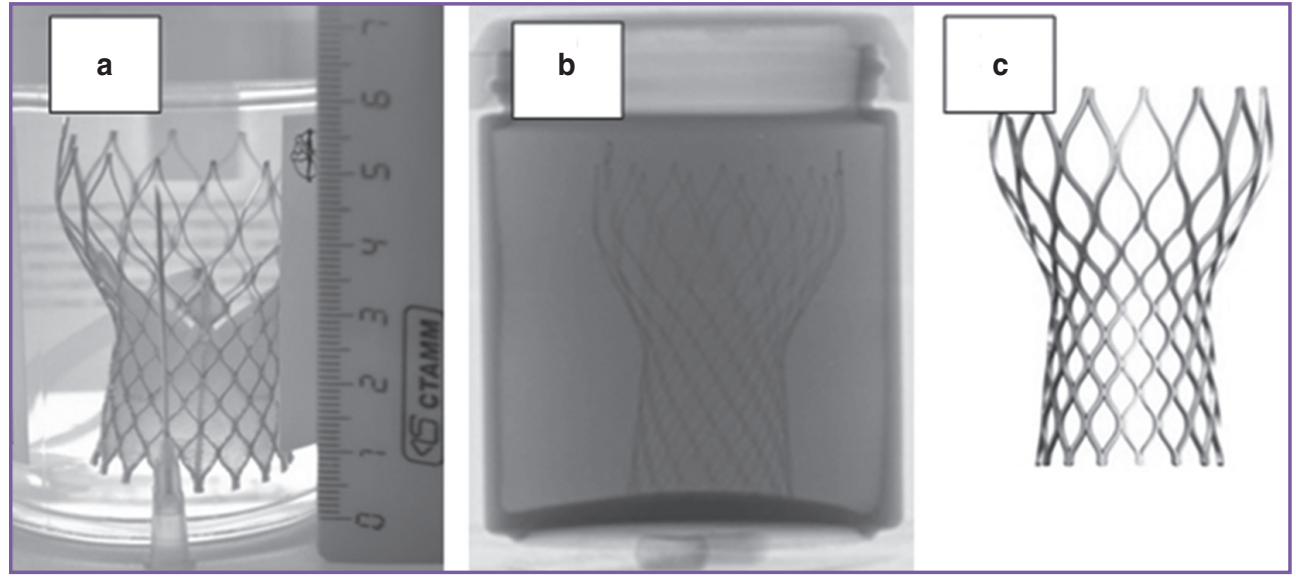

Figure 6. Building the model of bioprosthesis frame: (a) initial model in the container with a sterile fluid; (b) photo of the specimen projection; (c) a reconstructed model of the bioprosthesis frame

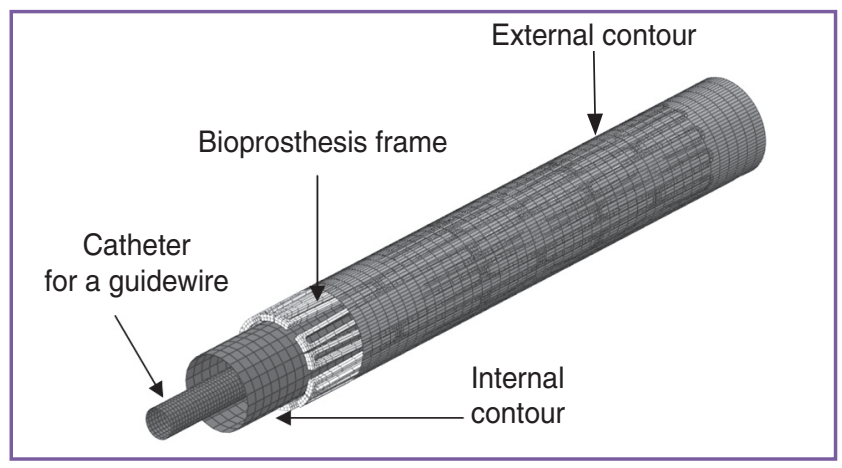

Figure 7. Simplified model of the delivery system and its components

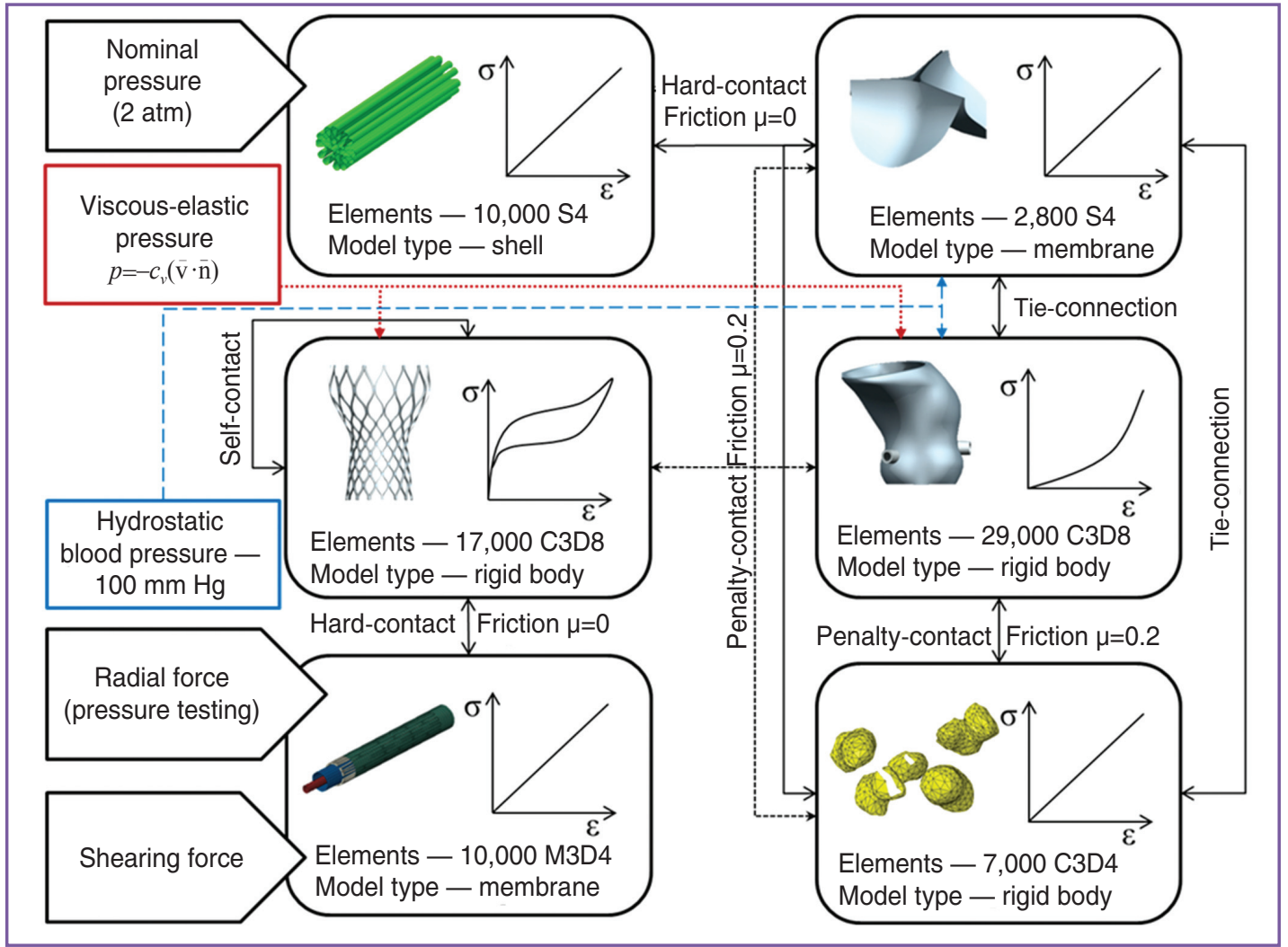

Figure 8. Model of the investigated biotechnical system 


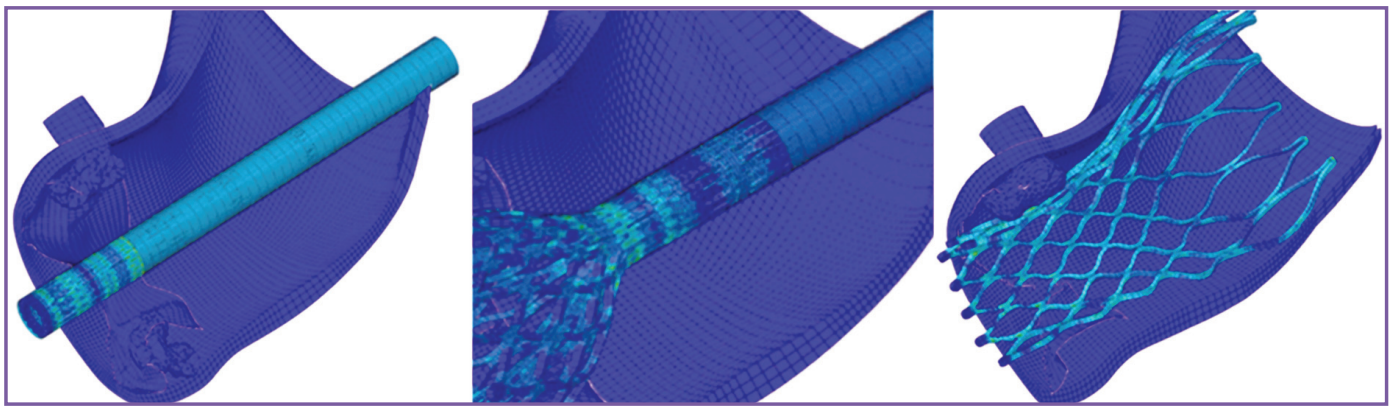

Figure 9. Stages of prosthesis implantation during computer modeling

Figure 10. Method of modeling error computation: $\mathrm{CM}$ - center of masses of each slice; $\mathrm{V}$ vector of the radial component (in the cylindrical coordinate system) for a point of MSCT data; $\delta-$ a modeling error for each point



leaflet apparatus was connected with aorta through the geometrical contact of grids with a rigidly pre-set distance between the nodes (tie-contact). To prevent interpenetration of the frame finite elements a selfcontact was used.

Simulation of interaction between the biotechnial system components with the help of FEM was performed as a series of successive steps: preliminary balloon dilatation - compression of the frame in the catheter release of the frame from the catheter (Figure 9).

Nonlinear material models were used to describe the root of the aorta (second order polynomial model [17]) and the stent (Auricchio's model [18]), as well as linear models for calcified fibrous annulus, leaflet apparatus $(E=22.6 \mathrm{MPa})$ [19] and for the models of calcium conglomerates ( $\mathrm{E}=10 \mathrm{MPa})$ [20].

An original algorithm using MATLAB R2014a (The MathWorks, USA) software was developed to assess the accuracy of the experiment results relative to clinical MSCT data of the patient with implanted bioprosthesis. Point arrays, corresponding to the center of the beam, obtained in 11 orthogonal frame slices according to the data of MSCT and FEM-modeling, were used as input data (Figure 10). The program algorithm searched the central axis and preliminary minimization of the rotation error of all slices relative each other, and calculated the center of slice masses as an average coordinate of all points. Then the program analyzed automatically each corrected slice separately, minimizing the distance between the corresponding points - a modeling error $\delta$. Based on the radial coordinate in the cylindrical coordinate system the program computed mean relative error ( $\delta$ rel) of the slice as a mean ratio of the modeling error $\delta$ to the length of vector $\mathrm{V}$ of the radial component (in the cylindrical coordinate system) for a point of MSCT data (See Figure 10). Also a total absolute error was computed for each of the 11 slices ( $\delta$ sum) as a sum of all modeling errors $\delta$ of the slice. Additionally, the program performed approximation of the FEM simulation data-set using canonical equation of the ellipse with the following calculation of the short and large elliptic radii, eccentricity (e) and ellipse area (S).

Results. In the course of the experiment it was found that the value of the total absolute error $\delta$ sum was essentially different in various slices. The greatest value was obtained in the frame zones directly contacting aorta - outflow (slice No.11 - $33.37 \mathrm{~mm}$ ) and inflow (slice No.2 - $35.07 \mathrm{~mm}$ ) (Figure 11). But when comparing the figures obtained with the initial "nonimplanted" geometry, the greatest discrepancy was noted in the outflow zone (slice No.11 - 40\%), the ratio of the total slice error to the sum of the radial component of the initial geometry amounted, on average, to $0.68 \pm 0.16$.

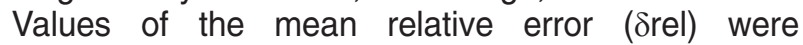
completely in agreement with the abovementioned results: the greatest deviation was registered in the 


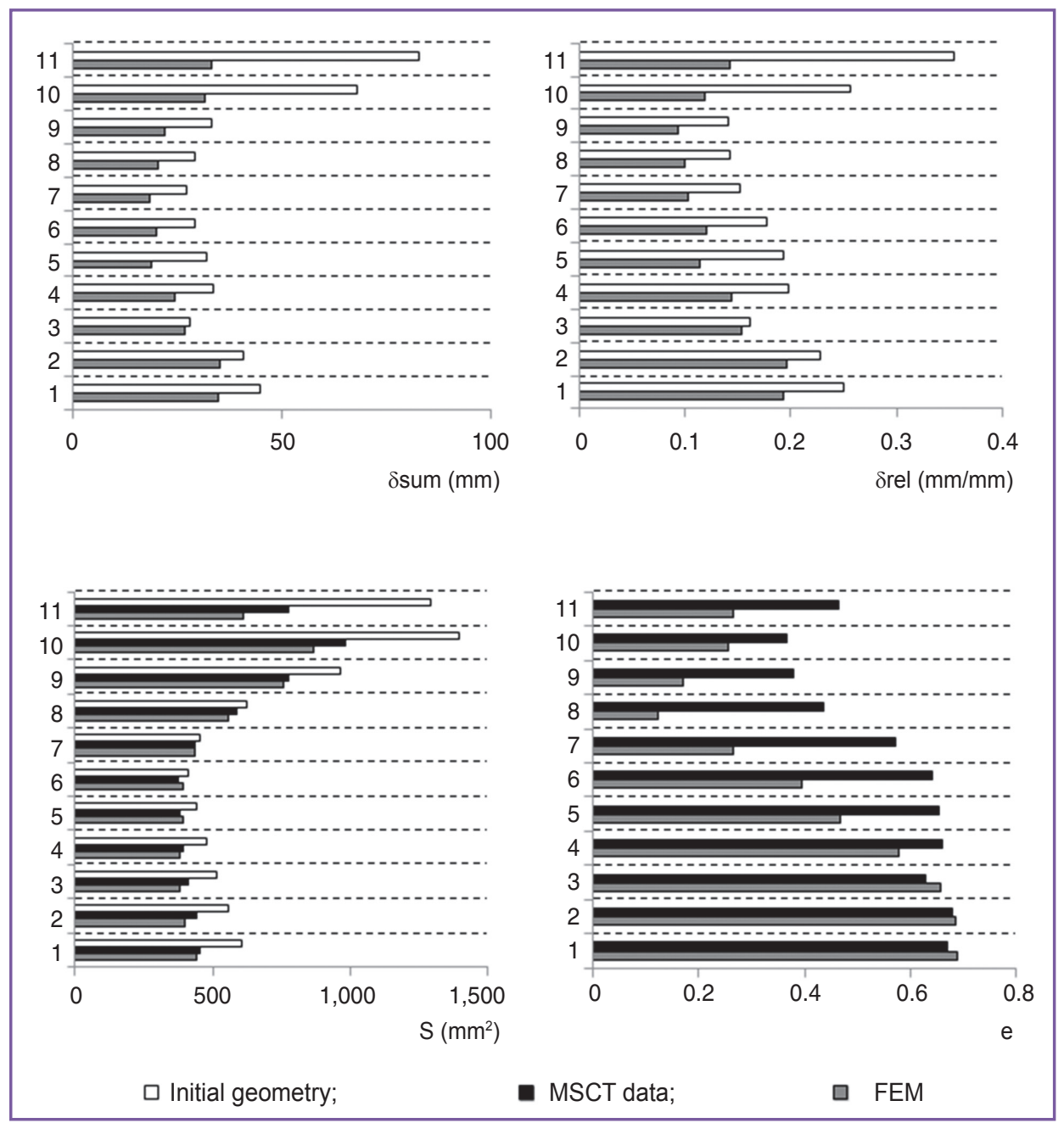

Figure 11. Results of error and quantitative characteristics analysis for valve prosthesis slices according to the data of FEM and MSCT: $\delta$ sum - total absolute and $\delta$ rel - mean relative error of a slice; $\mathrm{S}$ - area and e - eccentricity of approximating ellipse

inflow zone -19 and $20 \%$ for the first two slices, while the initial geometry differed by 25 and $23 \%$, respectively. Mean deviation for the slices was $12 \pm 4 \%$ in experiment.

In clinical practice measurement of eccentrcity (e) of the frame by slices is used as one of the ways for assessing the position of implanted transcatheter bioprostheses [21]. According to the previous investigations [22], this figure is of primary importance as a criterion of the leaflet apparatus performance and presence of regurgitation. In this investigation a high convergence of FEM and MSCT data could be achieved for the inflow zone (divergence of the simulation data according to the first three layers did not exceed $4 \%$ ), while the ellipticity of the middle and outflow zones, as FEM data showed, differed greatly from that of MSCT. The total error, determined in this way, was not greater than $26 \%$.

Another not less important parameter showing prosthesis implantation efficacy is an area of its annulus cross-section (S). This parameter characterizes indirectly valve throughput capacity and, upon the whole, allows the assessment of the implanted prosthesis size conformity. In the process of the study this parameter was distributed in accordance with the initial geometry of the prosthesis in the form of a "sand-glass": descending trend in the inflow and ascending in the outflow zones. The ratio of the annulus cross-section area of FEM prosthesis model according to FEM and MSCT data has demonstrated the best results of simulation assessment: more than $90 \%$ for the inflow and middle zones of the framework (slices No.1-8). Meanwhile, the differences with the initial "nonimplanted" geometry were also much more prominent compared to the other methods of simulation error assessment.

It is worth mentioning, that the last slice did not correspond to the described distribution, though, in general, it meets the characteristic behavior of the frame under considerable compression due to its specific geometry. In fact, the results of the investigation confirm the greatest radial displacement of the outflow zone of 


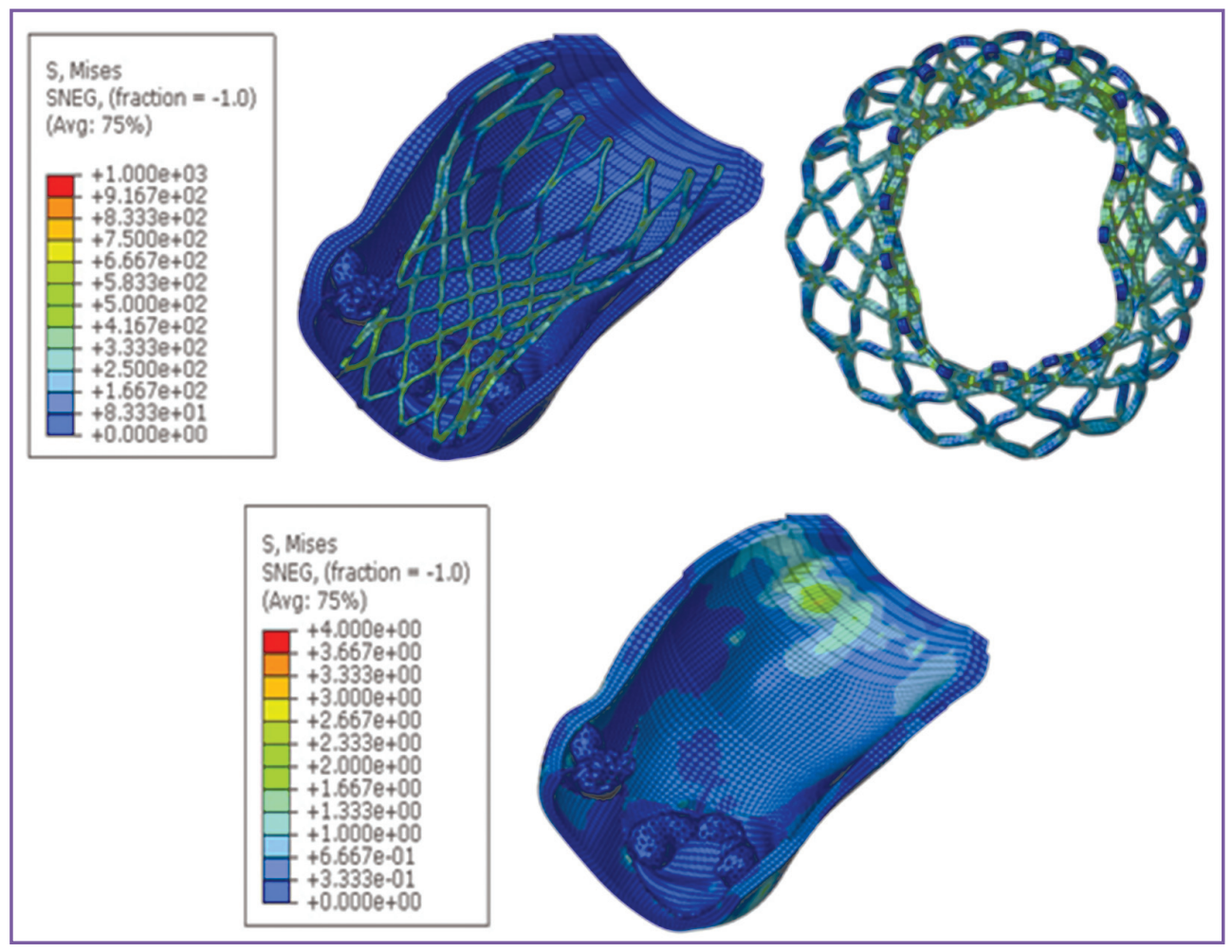

Figure 12. Von Mises stress distribution in the frame model (above) and aorta (below) as a result of their interaction

the frame, though when considering results together with stress distribution it is seen, that the zone of synotubular junction is subjected to the greaest stress. It speaks of the "anchor" function of the frame outflow zone (Figure 12).

Discussion. Russ et al. also assessed in their work [3] a total error of computer simulation and MSCT data using real implantation of CoreValve bioprosthesis. It has been proved, that application of complex nonlinear models of the material (hyperelasic) for the description of aortic root behavior, and also inclusion calcium conglomerates into calculations may reduce the simulation error to $5.5-4.2 \%$. In the present investigation


amounted to $12 \%$. The mean error value was $1.6 \mathrm{~mm}$, which, on the whole, corresponds to the resolution order of the tomograph used in this study $-0.625 \mathrm{~mm}$.

It should be mentioned, that in the investigation referred to [3], convergence of the FEM and MSCT results was evaluated only by a single parameter (similar to $\delta$ rel), which is considered by the authors of the present article to be insufficient. In this connection, a complex multicomponent approach to the verification of FEM data was applied, i.e. computation of errors within each slice and totally over the whole model, and the parameter of ellipticity as well.

Notably, that the greatest convergence of clinical MSCT and experimental FEM results, calculated by the sum of beam deviations, was obtained for the outflow zone of the bioprosthesis - the last two slices. This effect can be partly explained by a more simple geometry of the sinotubular junction zone. Thus, the result of simulation was determined by a single component only — the model material.

In spite of the fact, that the investigation was carried out on the basis of one clinical case, the developed method and a set of parameters studied can be used to solve the tasks of assessment the convergence of FEM simulation and clinical data.

Conclusion. Application of a higher level of detail, including simulation of calcium conglomerates and complex description of the system elements, make it possible to model implantation of transcatheter aortic vavular bioprosthesis with an accuracy depending on the tomograph resolution. The method of finite elements may be recommended for predicting outcomes of implanting minimally invasive devices.

Study Funding. The work was supported by the funds allocated to the Research Institute of Complex Problems of Cardiovascular Diseases.

Conflits of Interest. The authors have no conflicts of interest.

\section{References}

1. Mortier P., Holzapfel G.A., De Beule M., Van Loo D., Taeymans Y., Segers P., Verdonck P., Verhegghe B. A novel simulation strategy for stent insertion and deployment in curved coronary bifurcations: comparison of three drug-eluting stents. Ann Biomed Eng 2010; 38(1): 88-99, http://dx.doi.org/10.1007/ s10439-009-9836-5. 
2. Zajarias A., Cribier A.G. Outcomes and safety of percutaneous aortic valve replacement. J Am Coll Cardiol 2009; 53(20): 1829-1836, http://dx.doi.org/10.1016/j.jacc. 2008.11.059.

3. Russ C., Hopf R., Hirsch S., Sündermann S., Falk V., Székely G., Gessat M. Simulation of transcatheter aortic valve implantation under consideration of leaflet calcification. Conf Proc IEEE Eng Med Biol Soc 2013; 2013: 711-714, http://dx.doi. org/10.1109/EMBC.2013.6609599.

4. Généreux P., Head S.J., Hahn R., Daneault B., Kodali S., Williams M.R., van Mieghem N.M., Alu M.C., Serruys P.W., Kappetein A.P., Leon M.B. Paravalvular leak after transcatheter aortic valve replacement: the new Achilles' heel? A comprehensive review of the literature. J Am Coll Cardiol 2013; 61(11): 1125-1136, http://dx.doi.org/10.1016/ j.jacc.2012.08.1039.

5. Cribier A., Eltchaninoff $H_{\text {., }}$ Bash A., Borenstein N., Tron C., Bauer F., Derumeaux G., Anselme F., Laborde F., Leon M.B. Percutaneous transcatheter implantation of an aortic valve prosthesis for calcific aortic stenosis: first human case description. Circulation 2002; 106(24): 3006-3008, http://dx.doi. org/10.1161/01.cir.0000047200.36165.b8.

6. Buellesfeld L., Wenaweser P., Gerckens U., Mueller R., Sauren B., Latsios G., Zickmann B., Hellige G., Windecker S., Grube E. Transcatheter aortic valve implantation: predictors of procedural success - the Siegburg-Bern experience. Eur Heart J 2010; 31(8): 984-991, http://dx.doi.org/10.1093/eurheartj/ ehp570.

7. Ovcharenko E.A., Klyshnikov K.U., Glushkova T.V., Burago A.U., Zhuravleva I.U. Nonlinear isotropic material model of human aortic root. Tekhnologii zhivykh sistem 2014; 6: 43-47.

8. Ovcharenko E.A., Klyshnikov K.Y., Zhuravleva I.Y. Effect of the "3f enable" stent deformation on the hydrodynamic parameters. Klinicheskaya fiziologiya krovoobrashcheniya 2014; 2: 41-47.

9. Auricchio F., Conti M., Morganti S., Reali A. Simulation of transcatheter aortic valve implantation: a patient-specific finite element approach. Comput Methods Biomech Biomed Engin 2014; 17(12): 1347-1357, http://dx.doi.org/10.1080/10255842. 2012.746676.

10. Schultz C.J., Weustink A., Piazza N., Otten A., Mollet N., Krestin G., van Geuns R.J., de Feyter P., Serruys P.W., de Jaegere P. Geometry and degree of apposition of the CoreValve ReValving system with multislice computed tomography after implantation in patients with aortic stenosis. J Am Coll Cardiol 2009; 54(10): 911-918, http://dx.doi.org/10.1016/ j.jacc.2009.04.075.

11. Capelli G., Bosi G.M., Cerri E., Nordmeyer J., Odenwald T., Bonhoeffer P., Migliavacca F., Taylor A.M., Schievano S. Patient-specific simulations of transcatheter aortic valve stent implantation. Med Biol Eng Comput 2012; 50(2): 183-192, http://dx.doi.org/10.1007/s11517-012-0864-1.

12. Wang Q., Sirois E., Sun W. Patient-specific modeling of biomechanical interaction in transcatheter aortic valve deployment. J Biomech 2012; 45(11): 1965-1971, http://dx.doi. org/10.1016/j.jbiomech.2012.05.008.

13. Halevi R., Hamdan A., Marom G., Mega M., Raanani E., Haj-Ali R. Progressive aortic valve calcification: three-dimensional visualization and biomechanical analysis. J Biomech 2015; 48(3): 489-497, http://dx.doi.org/10.1016/j.jbiomech.2014.12.004.

14. Tzamtzis S., Viquerat J., Yap J., Mullen M.J., Burriesci G. Numerical analysis of the radial force produced by the MedtronicCoreValve and Edwards-SAPIEN after transcatheter aortic valve implantation (TAVI). Med Eng Phys 2013; 35(1): 125-130, http:// dx.doi.org/10.1016/j.medengphy.2012.04.009.

15. Li K., Sun W. Simulated thin pericardial bioprosthetic valve leaflet deformation under static pressure-only loading conditions: implications for percutaneous valves. Ann Biomed Eng 2010; 38(8): 2690-2701, http://dx.doi.org/10.1007/s10439010-0009-3.

16. Détaint D., Lepage L., Himbert D., Brochet E., MessikaZeitoun D., lung B., Vahanian A. Determinants of significant paravalvular regurgitation after transcatheter aortic valve: implantation impact of device and annulus discongruence. JACC Cardiovasc Interv 2009; 2(9): 821-827, http://dx.doi. org/10.1016/j.jcin.2009.07.003.

17. Morganti S., Conti M., Aiello M., Valentini A., Mazzola A., Reali A., Auricchio F. Simulation of transcatheter aortic valve implantation through patient-specific finite element analysis: two clinical cases. J Biomech 2014; 47(11): 2547-2555, http:// dx.doi.org/10.1016/j.jbiomech.2014.06.007.

18. Hamdan A., Guetta V., Konen E., Goitein O., Segev A., Raanani E., Spiegelstein D., Hay I., Di Segni E., Eldar M., Schwammenthal E. Deformation dynamics and mechanical properties of the aortic annulus by 4-dimensional computed tomography: insights into the functional anatomy of the aortic valve complex and implications for transcatheter aortic valve therapy. J Am Coll Cardiol 2012; 59(2): 119-127, http://dx.doi. org/10.1016/j.jacc.2011.09.045.

19. Wong D.T., Bertaso A.G., Liew G.Y., Thomson V.S., Cunnington M.S., Richardson J.D., Gooley R., Lockwood S., Meredith I.T., Worthley M.I., Worthley S.G. Relationship of aortic annular eccentricity and paravalvular regurgitation post transcatheter aortic valve implantation with CoreValve. J Invasive Cardiol 2013; 25(4): 190-195.

20. Kalejs M., von Segesser L.K. Rapid prototyping of compliant human aortic roots for assessment of valved stents. Interact Cardiovasc Thorac Surg 2009; 8(2): 182-186, http:// dx.doi.org/10.1510/icvts.2008.194134.

21. Baillargeon B., Rebelo N., Fox D.D., Taylor R.L., Kuhl E. The living heart project: a robust and integrative simulator for human heart function. Eur J Mech A Solids 2014; 48: 38-47, http://dx.doi.org/10.1016/j.euromechsol.2014.04.001.

22. Ovcharenko E.A., Klyshnikov K.U., Savrasov G.V., Nyshtaev D.V., Glushkova T.V. The choosing of optimal cell parameters of transcatheter aortic valve pros-thesis. Komp'yuternye issledovaniya i modelirovanie 2014; 6(6): 943-954. 